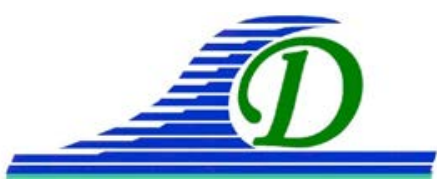

XIII ${ }^{\text {èmes }}$ Journées Nationales Génie Côtier - Génie Civil Dunkerque, 2-4 juillet 2014

DOI:10.5150/jngcgc.2014.116_ @ Editions Paralia CFL

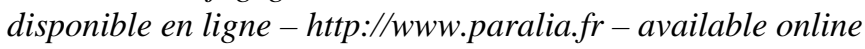

\title{
Extraction des métaux lourds des sédiments par méthode électrocinétique
}

\section{Yue SONG $^{1,2}$, Ahmed BENAMAR ${ }^{1}$, Salim MEZAZIGH ${ }^{2},{\text { Huaqing } \text { WANG }^{1}}^{1}$}

1. Université du Havre, UFR Sciences et Techniques, UMR 6294 CNRS - LOMC, 75 rue Bellot, CS 80540, 76058 Le Havre, France.

2. Université de Caen, Laboratoire Morphodynamique Continentale et Côtière, CNRSUMR 6143, 24 rue des Tilleuls, 14000 Caen, France.yue.song@univ-lehavre.fr

\section{Résumé :}

Chaque année, un volume considérable de sédiments de dragage est déposé dans des chambres de stockage en raison de leur contamination par les polluants organiques et/ou inorganiques. Plusieurs travaux scientifiques ont montré l'efficacité de la méthode de traitement électrocinétique pour la décontamination des sédiments. Cette technologie est prometteuse malgré les difficultés rencontrées au cours des traitements en raison notamment des propriétés physico-chimiques des sédiments (faible perméabilité, pollution multiple, forte teneur en eau, etc.). Elle nécessite alors l'adaptation des électrolytes en fonction des éléments polluants présents. L’étude expérimentale menée a consisté dans un premier temps à utiliser comme additifs dans le processus d'électromigration l'eau distillée comme solution anodique et l'acide citrique comme solution cathodique. Différentes valeurs du champ électrique ont été appliquées (0,5 $\mathrm{V} \mathrm{cm}^{-1}, 1,0 \mathrm{~V} \mathrm{~cm}^{-1}, 1,5 \mathrm{~V} \mathrm{~cm}^{-1}$ ) pour évaluer l'influences du paramètre électrique sur les débits électro-osmotiques, sur l'abattement de la pollution et enfin, sur l'efficacité énergétique d'abattement de différents métaux (As, $\mathrm{Cd}, \mathrm{Cr}, \mathrm{Cu}, \mathrm{Ni}, \mathrm{Pb}, \mathrm{Zn}$ et $\mathrm{Hg}$ ).

Mots-clés : Sédiment, Dragage, Electrocinétique, Métaux lourds.

Abstract: Every year, a considerable volume of dredged sediments is deposited in storage sites because of their contamination by organic and/or inorganic pollutants. Recent studies have demonstrated the efficiency of the electrokinetic decontamination method of heavy metals contained in dredged sediments. Despite the difficulties encountered during treatment, which are due to the physical and chemical properties of dredged sediments (low permeability, several interacting pollutants, high moisture content, etc.) electrokinetic remediation is a promising method. It is so necessary to adapt an electrolyte solution to targeted pollutants. The experimental study was carried out using distilled water and citric acid as the anode and the cathode solutions respectively. Different electrical potentials were applied $\left(0.5 \mathrm{~V} \mathrm{~cm}^{-1}, 1.0 \mathrm{~V} \mathrm{~cm}\right.$, $1.5 \mathrm{~V} \mathrm{~cm}^{-1}$ ) in order to assess their influence on the electro-osmotic flow, the metal removal and finally on energy consumption as regards to removal ratio of metals pollution (As, Cd, Cr, Cu, Ni, Pb, Zn and $\mathrm{Hg}$ ).

Keywords: Sediment, Dredging, Electrokinetic, Heavy metals. 


\section{Introduction}

Afin d'assurer la sécurité de la navigation en maintenant la profondeur des ports et des chenaux, 55 millions de mètres cubes de sédiment sont dragués chaque année dans le territoire français (BENARD, 2009). En raison des recommandations des conventions internationales OSPAR et de Barcelone, Le Groupe d'Étude et d'Observation sur le Dragage et L'Environnement (GEODE) a proposé les niveaux de références N1 et N2 pour servir de références dans les procédures d'instruction des autorisations de dragage et d'immersion (LE GUYADER, 2012). Les niveaux GEODE concernent les contaminants inorganiques comme les éléments-traces métalliques (ETM) et les contaminants organiques (PCB, TBT et HAP). Les sédiments dragués et déposés à terre sont considérés comme des déchets. Dans ce cas, ces sédiments doivent respecter la directive européenne <Décision européenne 2003/33/CE (JOCE nº 11 16/01/2003)>.

En raison des problèmes économiques et environnementaux engendrés par le stockage des sédiments, des solutions alternatives de décontamination et de valorisation des sédiments sont fortement recherchées. A cause d'une faible perméabilité et d'une grande surface spécifique, les sédiments sont relativement résistants au traitement avec des techniques traditionnelles (GENC et al., 2008). Néanmoins, plusieurs travaux ont montré l'efficacité de la méthode de traitement électrocinétique pour la décontamination des sédiments (AMMAMI et al., 2013 ; YEUNG \& GU, 2011). L'objectif principal de l'électrocinétique est d'obtenir la migration des contaminants sous un champ électrique appliqué via l'électro-osmose, l'électromigration et l'électrophorèse (VIRKUTYT et al., 2002).

La migration des ions $\mathrm{H}+$ peut mobiliser les métaux lourds dans les sédiments afin d'accélérer leur migration. Par contre, les ions $\mathrm{OH}$ - ont une action opposée. Ils peuvent combiner les ions métalliques lourds et les précipiter dans les sédiments. Toutefois, plusieurs travaux démontrent qu'un contrôle du pH (par ajout d'acide) à la cathode peut neutraliser les ions $\mathrm{OH}$ - et améliorer la teneur en métaux lourds en électromigration dans les sédiments pollués (GU \& YEUNG, 2011 ; YUAN \& CHIANG, 2008 ; YEUNG \& GU, 2011 ; PAZOS et al., 2006 ; AMMAMI et al., 2013).

L’objectif de ce travail est l'étude de l'efficacité de la méthode électrocinétique pour l'extraction des métaux lourds des sédiments en utilisant différentes valeurs du champ électrique dans l'objectif de déterminer l'effet de ce paramètre sur l'abattement des ETM et sur l'efficacité énergétique.

\section{Matériels et méthodes}

\subsection{Les sédiments}

Les échantillons de sédiments de dragage ont été prélevés en juillet 2010 à l'aide d'une pelle dans la chambre de dépôt Tancarville. Puis, les échantillons prélevés sont conservés dans un conteneur étanche et stockés dans une enceinte réfrigérée à $4{ }^{\circ} \mathrm{C}$ afin 


\section{XIII ${ }^{\text {èmes }}$ Journées Nationales Génie Côtier - Génie Civil \\ Dunkerque, 2-4 juillet 2014}

de réduire les actions d'auto-dégradation des ETM et de la Matière Organique. Les résultats de la granulométrie laser (Multisizer 2000 - Malvern) effectuée sur ce sédiment montrent que $94 \%$ des particules sont des fines $(<80 \mu \mathrm{m})$, dont $6,3 \%$ de particules classés dans la fraction argileuse $(\mathrm{d}<2 \mu \mathrm{m}), 86,2 \%$ de fraction limoneuse $(2 \mu \mathrm{m}<\mathrm{d}<63 \mu \mathrm{m})$ et $7,5 \%$ de fraction sableuse $(\mathrm{d}>63 \mu \mathrm{m})$. La valeur de l'essai au bleu de Méthylène (NF P 94-068) est 4,8 \pm 0,2, indiquant que le sédiment peut être classé dans la fraction limoneux-argileuse.

L'opération de minéralisation a été réalisée par notre soin au laboratoire à l'aide de l'appareil micro-onde de minéralisation (Discover SP D, CEM $\mu$-waves), selon le protocole suivant : la fraction solide $(0,5 \mathrm{~g})$ est attaquée par une solution acide d'un volume de $9 \mathrm{ml}$ ( $6 \mathrm{ml}$ d'acide nitrique et $2 \mathrm{ml}$ d'acide chlorhydrique), puis chauffée à $150{ }^{\circ} \mathrm{C}$ pendant 504 heures. Après un refroidissement à $60^{\circ} \mathrm{C}$, la solution est filtrée à 0,45 $\mu \mathrm{m}$ à l'aide d'une pompe à vide. La solution est ensuite diluée à $30 \mathrm{ml}$ dans l'eau ultra-pure puis conservée dans un flacon étanche et stockée dans un réfrigérateur à $4{ }^{\circ} \mathrm{C}$. Les analyses des solutions ont été faites par ICP-AES (laboratoire COBRA-INSA de Rouen). Les résultats d'analyse des sédiments bruts et la comparaison avec les niveaux GEODE et la réglementation Européenne sont montrés dans le tableau 1.

Tableau 1. Résultats d'analyses chimiques du sédiment de Tancarville

\begin{tabular}{|c|c|c|c|c|c|c|c|c|}
\hline & As $(\mathrm{mg} / \mathrm{kg})$ & $\mathrm{Cd}(\mathrm{mg} / \mathrm{kg})$ & $\mathrm{Cr}(\mathrm{mg} / \mathrm{kg})$ & $\mathrm{Cu}(\mathrm{mg} / \mathrm{kg})$ & $\mathrm{Hg}(\mathrm{g} / \mathrm{kg})$ & $\mathrm{Ni}(\mathrm{mg} / \mathrm{kg})$ & $\mathrm{Pb}(\mathrm{mg} / \mathrm{kg})$ & $\mathrm{Zn}(\mathrm{mg} / \mathrm{kg})$ \\
\hline $\mathrm{N} 1^{1}$ & 25 & 1,2 & 90 & 45 & 0,4 & 100 & 37 & 276 \\
\hline $\mathrm{N}^{1}$ & 50 & 2,4 & 180 & 90 & 0,8 & 200 & 74 & 552 \\
\hline Inertes $^{2}$ & 0,5 & 0,04 & 0,5 & 2 & 0,01 & 0,4 & 0,5 & 4 \\
\hline $\begin{array}{c}\text { Non } \\
\text { dangereux }^{2}\end{array}$ & 2 & 1 & 10 & 50 & 0,2 & 10 & 10 & 50 \\
\hline Dangereux $^{2}$ & 25 & 5 & 70 & 100 & 2 & 40 & 50 & 200 \\
\hline Cobra & $14,95 \pm 1,95$ & $4,6 \pm 0,7$ & $136,34 \pm 24$ & $63,97 \pm 12$ & $2,01 \pm 0,62$ & $38,97 \pm 1,6$ & $63,93 \pm 18$ & $222,8 \pm 77,2$ \\
\hline
\end{tabular}

${ }^{1}$ Valeurs guides des niveaux 1 et 2 pour les Métaux GEODE (Arrêté du 14/06/2000)

${ }^{2}$ Valeurs limites des critères et des procédures d'admission des déchets dans les décharges

< Directive européenne 2003/33/CE (JOCE nL11 16/01/2003)>

\subsection{Dispositif expérimental}

Le dispositif expérimental de l'extraction des métaux lourds est présenté sur la figure 1. Le montage principal est une cellule en Téflon-PTFE. Elle est constituée de 3 parties : un compartiment central de forme cylindrique (longueur $\times$ diamètre intérieur $=$ $14 \mathrm{~cm} \times 4,9 \mathrm{~cm}$ ) et deux compartiments latéraux aménagés pour recevoir les électrodes et le réactif. Une masse d'environ 380 g de sédiment homogène, avec une teneur en eau voisine de 95\%, est introduite dans le compartiment central. Trois orifices de dégazage sont percés en ligne sur la paroi du compartiment central et sont fermés par 3 bouchons. A chaque extrémité du compartiment central est placé un papier filtre $(0,45 \mu \mathrm{m})$ et une grille. L'ensemble des compartiments est assemblé à l'aide de 4 tiges de serrage. 


\section{Thème 8 - Gestion et valorisation des sédiments marins}

L'étanchéité est assurée grâce à 2 joints placés entre chacune des grilles et le compartiment d'électrode. L'anolyte et le catholyte sont acheminés dans les compartiments d'électrodes par le fond des compartiments à l'aide de deux pompes péristaltiques (03S STEPDOS-KNFlab) à un débit constant de $5 \mathrm{ml} / \mathrm{h}$. L'eau distillée est choisie comme anolyte et comme catholyte, l'acide citrique, pour sa propriété biodégradable et non-nuisible pour l'environnement, est utilisé à une concentration de $1 \mathrm{~mol} / \mathrm{L}$. Les effluents sont récupérés par les trop-pleins des compartiments d'électrodes et conservés dans des flacons. Les électrodes en graphite sont introduites dans les compartiments d'électrodes et immergées dans les réactifs. Egalement, les gaz produits par les réactions d'électrolyse peuvent s'échapper par le haut des compartiments. Une tension électrique constante est appliquée entre les électrodes à l'aide d'un générateur de courant continu, et l'intensité électrique est mesurée à l'aide un ampèremètre.

Trois essais avec des valeurs de potentiel électrique différentes $\left(0,5 \mathrm{~V} \mathrm{~cm}^{-1}, 1,0 \mathrm{~V} \mathrm{~cm}{ }^{-1}\right.$ et $1,5 \mathrm{~V} \mathrm{~cm}^{-1}$ ) ont été réalisés, avec une durée 21 jours pour chaque essai. Au cours des essais, la variation de l'intensité électrique ainsi que les volumes et le $\mathrm{pH}$ des effluents de l'anode et de la cathode sont mesurés régulièrement. A la fin des essais, la matrice des sédiments est découpée en 4 tranches de même épaisseur de l'anode à la cathode et sont analysées pour déduire la concentration en métaux lourds.

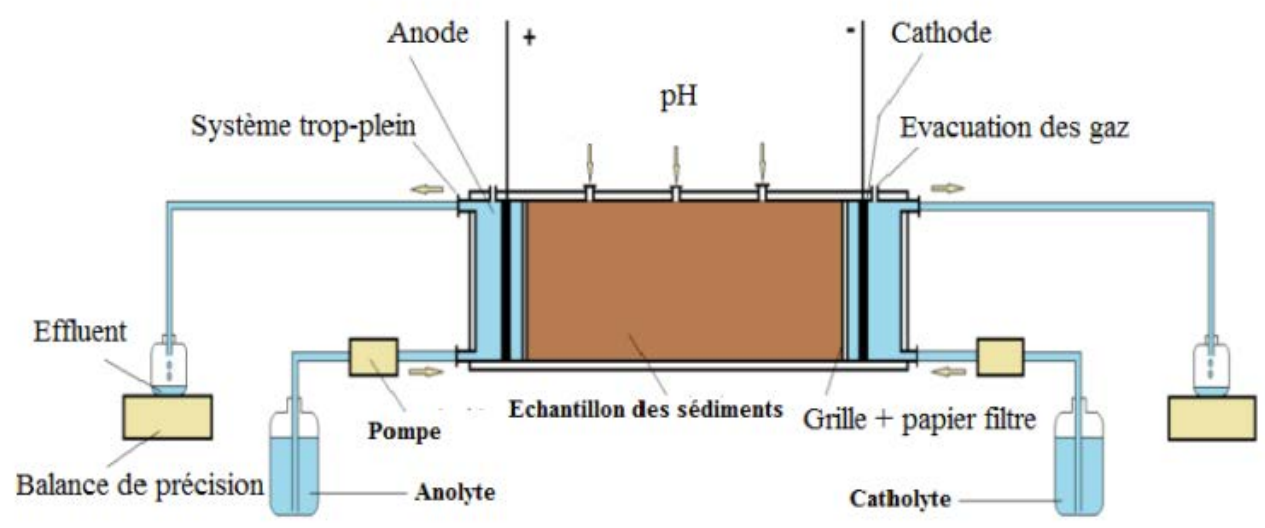

Figure 1. Schéma du dispositif expérimental.

\section{Résultats et Interprétation}

\section{1 Évolution du débit électroosmotique}

Chaque jour, les débits électroosmotiques entre l'anode et la cathode ont été calculés par la différence entre les débits entrant et sortant. Les débits d'électro-osmose moyens, $Q_{e}$ $\left(\mathrm{ml} \mathrm{h}^{-1}\right)$, à l'anode $\left(Q_{e}(a)\right)$ et à la cathode $\left(Q_{e}(k)\right)$ sont rapportés sur le tableau 2. Le $Q_{e}$ négatif mesuré correspond à une inversion du débit électroosmotique qui pourrait être 


\section{XIII ${ }^{\text {èmes }}$ Journées Nationales Génie Côtier - Génie Civil \\ Dunkerque, 2-4 juillet 2014}

néfaste pour l'élimination des polluants, phénomène déjà observé par AMMAMI et al. (2013). D'autre part, on remarque que le débit d'électro-osmose à la cathode est supérieur à celui de l'anode, ce qui est normal car dans le cas contraire, on se retrouverait dans une situation d'un flux négatif. Le débit moyen augmente avec le potentiel électrique appliqué car $Q_{e}$ dans une cellule est directement proportionnel à la valeur du champ électrique appliqué $\left(i_{e}=\mathrm{V} \mathrm{cm}^{-1}\right)$ (YUAN \& CHIANG, 2008) :

$Q_{e}=K_{e} i_{e} A$

où $A\left(\mathrm{~cm}^{2}\right)$ est la section de la cellule et $K_{e}$ est le coefficient de perméabilité électroosmotique $\left(\mathrm{cm}^{2} \mathrm{~V}^{-1} \mathrm{~S}^{-1}\right)$. Le calcul de ces coefficients à l'anode $\left(K_{e}(a)\right)$ et à la cathode $\left(K_{e}(k)\right)$ donne les valeurs présentées sur le tableau 2.

Tableau 2. Débits électroosmotiques moyens et perméabilité électroosmotique

\begin{tabular}{lllll}
\hline$i_{e}\left(\mathrm{~V} \mathrm{~cm}^{-1}\right)$ & $Q_{e}(a)\left(\mathrm{ml} \mathrm{h}^{-1}\right)$ & $Q_{e}(\mathrm{k})\left(\mathrm{ml} \mathrm{h}^{-1}\right)$ & $K_{e}(a)\left(\mathrm{cm}^{2} \mathrm{~V}^{1} \mathrm{~S}^{-1}\right)$ & $K_{e}(\mathrm{k})\left(\mathrm{cm}^{2} \mathrm{~V}^{1} \mathrm{~S}^{-1}\right)$ \\
\hline 0,5 & $-0,16$ & 0,69 & $-4,6410^{-6}$ & $20,3810^{-6}$ \\
1,0 & 1,16 & 1,80 & $17,0510^{-6}$ & $26,5410^{-6}$ \\
1,5 & 1,98 & 2,27 & $19,5410^{-6}$ & $22,3510^{-6}$ \\
\hline
\end{tabular}

\subsection{Abattement des concentrations en métaux lourds dans le sédiment}

A la fin des essais, les effluents recueillis dans les compartiments anodique et cathodique ainsi que les fractions restant dans le sédiment sont analysés par le protocole de la minéralisation de sédiment (voir le paragraphe 2.1). Les valeurs des abattements des ETM sont illustrées sur la figure 2.

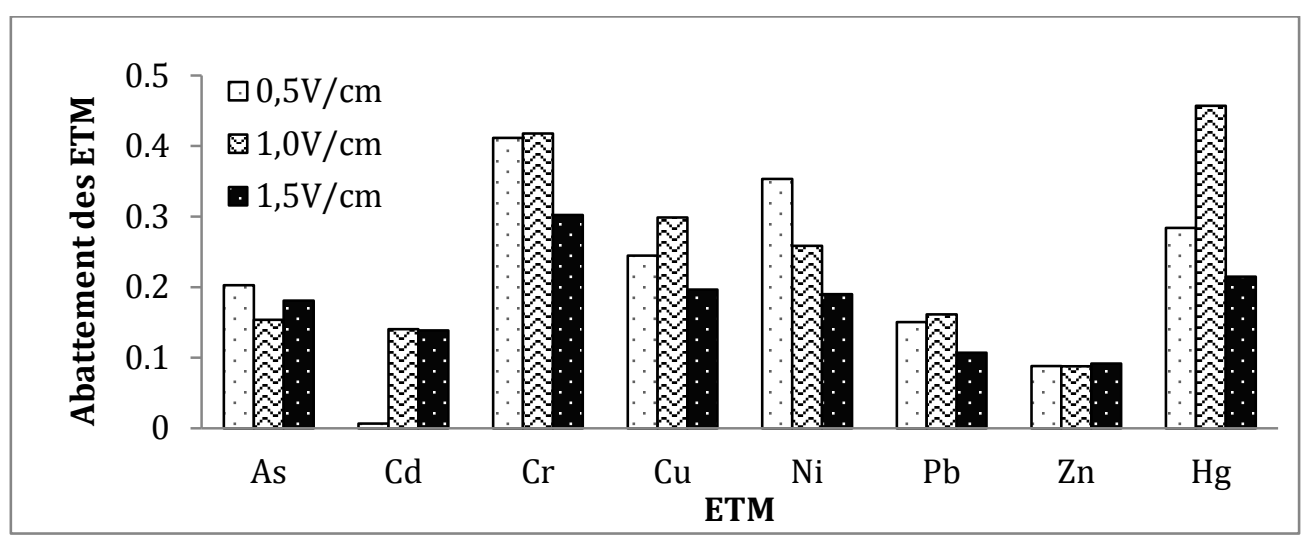

Figure 2. Abattement moyen des ETM dans le sédiment de Tancarville pour les différents traitements électrocinétiques.

L'ordre d'abattement des ETM est représenté par les valeurs données dans le Tableau 3. Globalement, nous avons observé que notre traitement électrocinétique est plus efficace pour les métaux $\mathrm{Cr}$, $\mathrm{Ni}$, Cu et $\mathrm{Hg}$ que pour les autres métaux (notamment $\mathrm{Zn}$ et $\mathrm{Cd}$ ) qui sont plus difficiles à extraire. Pour mettre en évidence l'effet du potentiel électrique, nous pouvons comparer les abattements par type d'ETM. Nous observons que quand le 
potentiel électrique est élevé $\left(1,5 \mathrm{~V} \mathrm{~cm}^{-1}\right)$, les abattements des ETM ne sont pas les plus importants. Par contre, à faible potentiel $\left(0,5 \mathrm{~V} \mathrm{~cm}^{-1}\right)$, l'abattement est plus élevé pour As et Ni. Par ailleurs, les abattements sont plus élevés pour $\mathrm{Cr}, \mathrm{Cu}, \mathrm{Pb}$ et $\mathrm{Hg}$ avec le potentiel de $1,0 \mathrm{~V} \mathrm{~cm}^{-1}$. Il y a très peu d'abattement pour le $\mathrm{Cd}$ avec le potentiel de $0,5 \mathrm{~V} \mathrm{~cm}^{-1}$. Le fractionnement des ETM a été rapporté par TESSIER et al. (1979) : cinq fractions sont classées par ordre de difficulté pour extraire, la fraction "Échangeable", la fraction "Carbonate", la fraction "Oxydes", la fraction "Sulfure et organique" et la fraction "Résiduels". D’après ZHOU et al. (2005), Zn est un type d’ETM qui se transforme facilement de fraction "échangeable" vers la fraction "résiduels" et devient alors plus difficile à extraire durant les procédés de traitement électrocinétique. Donc, l'abattement de Zn est non seulement faible, mais aussi ne varie pas sensiblement en fonction de la valeur du potentiel électrique.

La figure 3 présente l'efficacité énergique des abattements des ETM dans les sédiments de Tancarville. Il est indiqué les consommations d'énergie pour abaisser de $1 \%$ la concentration des ETM par tonne de sédiment sec. Sachant que la consommation d'énergie augmente avec le potentiel électrique, on peut déduire que l'efficacité énergique n’augmente pas avec l'augmentation du potentiel électrique.

Tableau 3. Classement des abattements des ETM dans les sédiments de Tancarville par rapport aux différents potentiels.

\begin{tabular}{ll}
\hline $0,5 \mathrm{~V} . \mathrm{cm}^{-1}$ & $\mathrm{Cr}>\mathrm{Ni}>\mathrm{Hg}>\mathrm{Cu}>\mathrm{As}>\mathrm{Pb}>\mathrm{Zn}>\mathrm{Cd}$ \\
$1,0 \mathrm{~V} . \mathrm{cm}^{-1}$ & $\mathrm{Hg}>\mathrm{Cr}>\mathrm{Cu}>\mathrm{Ni}>\mathrm{Pb}>\mathrm{As}>\mathrm{Cd}>\mathrm{Zn}$ \\
$1,5 \mathrm{~V} . \mathrm{cm}^{-1}$ & $\mathrm{Cr}>\mathrm{Hg}>\mathrm{Cu}>\mathrm{Ni}>\mathrm{As}>\mathrm{Cd}>\mathrm{Pb}>\mathrm{Zn}$ \\
\hline
\end{tabular}

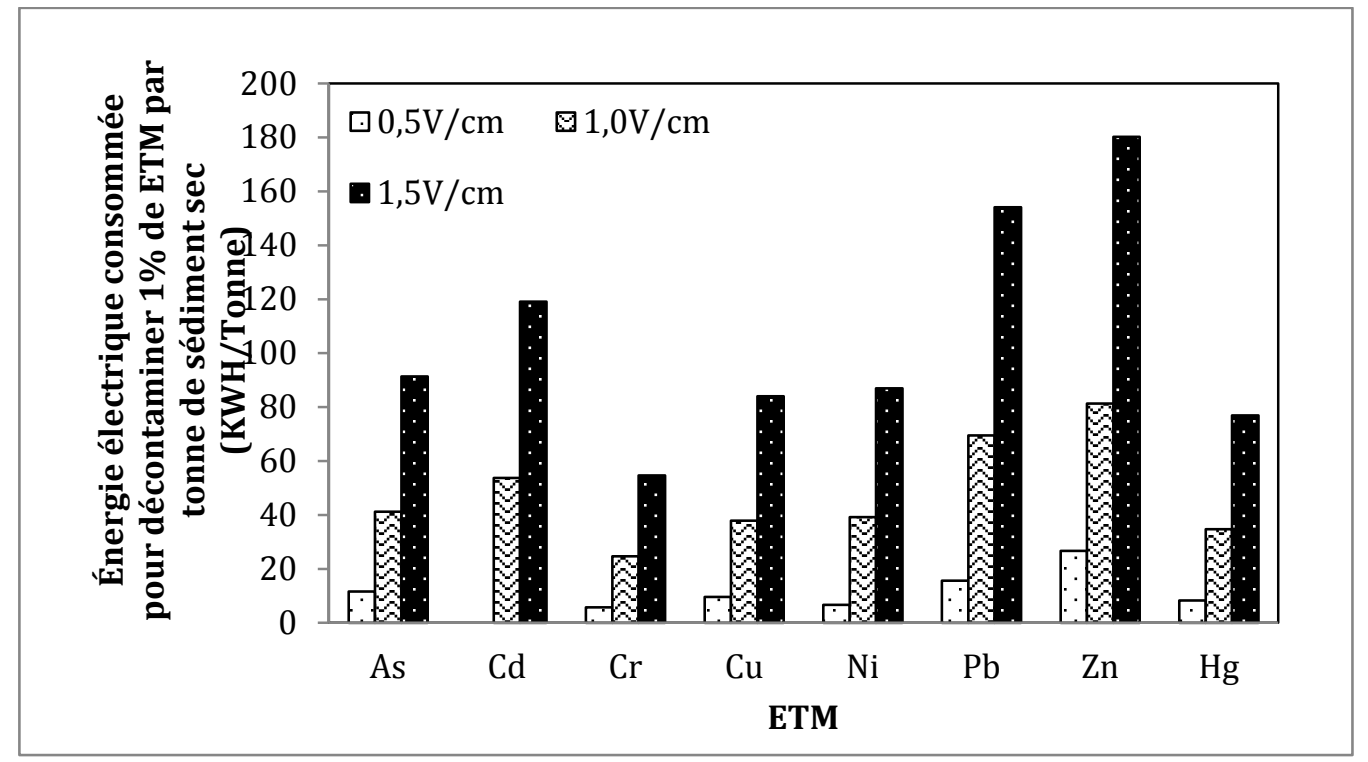

Figure 3. Efficacité énergétique d'abattement des ETM. 


\section{XIII ${ }^{\text {èmes }}$ Journées Nationales Génie Côtier - Génie Civil \\ Dunkerque, 2-4 juillet 2014}

\section{Conclusion}

L'objet de ce travail consiste à étudier l'efficacité de décontamination des sédiments pollués par les métaux lourds par le procédé électrocinétique. L'utilisation de l'acide citrique comme catholyte a montré une activation performante et durable du processus d'électromigration. Les résultats ont montré une augmentation du débit électroosmotique en fonction du potentiel électrique appliqué. Le couplage de l'eau distillée et de l'acide citrique comme solution anodique et cathodique, respectivement, a été efficace dans l'extraction des ETM Cr et Hg. Les résultats ont démontré qu'un faible potentiel électrique peut non seulement présenter une assez bonne efficacité de décontamination des ETM, mais également, il peut réduire la consommation d'énergie. Un compromis devra être trouvé entre le niveau d'abattement des ETM et la consommation d'énergie. L'activation alternée de la charge électrique présente une alternative intéressante pour le maintien d'un niveau d'électromigration des ETM et l'économie d'énergie.

\section{Références}

AMMAMI M.T. (2013). Contribution à l'étude des processus électrocinétiques appliqués aux sédiments de dragage. Thèse de l’Université du Havre. 186 p.

AMMAMI M.T., BENAMAR A., WANG H.Q., BAILLEUL C., LEGRAS M., LE DERF F., PORTET-KOLTALO F. (2013). Simultaneous electrokinetic removal of polycyclic aromatic hydrocarbons and metals from a sediment using mixed enhancing agents. Int. J. of Environmental Science and Technology. http://dx.doi.org/10.1007/s13762-013-0395-9 BENARD A. (2009). Impacts sur les milieux aquatiques des sédiments de dragage gérés à terre: Problématique, contexte réglementaire, modélisation du transfert de contaminants organiques. Rapport INERIS $n^{\circ} 24$ (partie 2) et $n^{\circ} 25.64 \mathrm{p}$

GENC A., CHASE G., FOOS A. (2008). Electrokinetic removal of manganese from river sediment. Water, Air, and Soil Pollution, Vol. 197(1-4), pp 131-141. http://dx.doi.org/10.1007/s11270-008-9796-7

GU Y.-Y., YEUNG A.T. (2011). Desorption of cadmium from a natural Shanghai clay using citric acid industrial wastewater. Journal of Hazardous Materials, Vol. 191(1-3), pp 144-149. http://dx.doi.org/10.1016/j.jhazmat.2011.04.054

LE GUYADER C. (2012). Enquête "Dragage 2009" synthèse des données. RapportCETMEF 24 p.

NF P 94-068 - Sols : Reconnaissance et essais Mesure de la capacité d'adsorption de bleu de méthylène d'un sol ou d'un matériau rocheux.

PAZOS M., SANROMAN M.A., CAMESELLE C. (2006). Improvement in electrokinetic remediation of heavy metal spiked kaolin with the polarity exchange technique. Chemosphere, Vol. 62(5), pp 817-22. http://dx.doi.org/10.1016/j.chemosphere.2005.04.071

TESSIER A., CAMPBELL P.G.C., BISSON M. (1979). Sequential extraction procedure for the speciation of particulate trace metals. Analytical Chemistry, Vol. 
Thème 8 - Gestion et valorisation des sédiments marins

51(7): pp 844-851. http://pubs.acs.org/doi/abs/10.1021/ac50043a017

VIRKUTYT J., SILLANPÄÄ M., LATOSTENMAA P. (2002). Electrokinetic soil remediation--critical overview. The Science of the total environment, Vol. 289(1-3), pp 97-121. http://dx.doi.org/10.1016/S0048-9697(01)01027-0

YEUNG A.T., GU Y. (2011). A review on techniques to enhance electrochemical remediation of contaminated soils. Journal of Hazardous Materials, Vol. 195, pp 11-29. http://dx.doi.org/10.1016/j.jhazmat.2011.08.047

YUAN C., CHIANG T. (2008). Enhancement of electrokinetic remediation of arsenic spiked soil by chemical reagents. Journal of Hazardous Materials, Vol. 152(1), pp 309-315. http://dx.doi.org/10.1016/j.jhazmat.2007.06.099

ZHOU D., DENG C.-F., CANG L., ALSHAWABKEH A.N. (2005). Electrokinetic remediation of a $\mathrm{Cu}-\mathrm{Zn}$ contaminated red soil by controlling the voltage and conditioning catholyte pH. Chemosphere, Vol. 61(4), pp 519-527. http://dx.doi.org/10.1016/j.chemosphere.2005.02.055 